\title{
Intermediate-band Surface Photometry of the Edge-on Galaxy: NGC 4565
}

\author{
Hong $\mathrm{Wu}^{1}$, David Burstein ${ }^{2}$, Zugan Deng ${ }^{1,3}$, Xu Zhou ${ }^{1}$, Zhaohui Shang ${ }^{4}$, Zhongyuan Zheng ${ }^{1}$, Jiansheng \\ Chen $^{1}$, Hongjun $\mathrm{Su}^{1}$, Rogier A. Windhorst ${ }^{2}$, Wen-ping Chen ${ }^{5}$, Zhenlong Zou ${ }^{1}$, Xiaoyang Xia ${ }^{1,6}$, Zhaoji \\ Jiang $^{1}$, Jun $\mathrm{Ma}^{1}$, Suijian Xue ${ }^{1}$, Jin Zhu ${ }^{1}$, Fuzhen Cheng ${ }^{7,1}$, Yong-Ik Byun ${ }^{5,8}$, Rui Chen ${ }^{1}$, Licai Deng ${ }^{1}$, \\ Xiaohui Fan ${ }^{9}$, Li-Zhi Fang ${ }^{10}$, Xu Kong ${ }^{7,1}$, Yong Li $^{2}$, Weipeng Lin ${ }^{1,11}$, Phillip Lu ${ }^{12}$, Wei-hsin Sun ${ }^{5}$, \\ Wean-shun Tsay ${ }^{5}$, Wen $\mathrm{Xu}^{2}$, Haojing $\mathrm{Yan}^{2}$, and Zheng Zheng ${ }^{13}$
}

Received —

${ }^{1}$ National Astronomical Observatories, Chinese Academy of Sciences, Beijing 100012, China

${ }^{2}$ Department of Physics and Astronomy, Box 871504, Arizona State University, Tempe, AZ 85287-1504

${ }^{3}$ Graduate School, Chinese Academy of Sciences, Beijing 100080, China

${ }^{4}$ Department of Astronomy, University of Texas at Austin, Austin, TX 78712

${ }^{5}$ Institute of Astronomy, National Central University, Chung-Li, Taiwan, China

${ }^{6}$ Department of Physics, Tianjin Normal University, China

${ }^{7}$ Center for Astrophysics, University of Science and Technology of China, Hefei, 230026, China

${ }^{8}$ Center for Space Astrophysics and Department of Astronomy, Yonsei University, Seoul, 120-749, Korea

${ }^{9}$ Princeton University Observatory, Princeton, New Jersey, 08544

${ }^{10}$ Department of Physics, University of Arizona, Tucson, AZ 85721

11 The Partner Group of MPI für Astrophysik, Shanghai Astronomical Observatory, Shanghai 200030, China

${ }^{12}$ Department of Physics and Astronomy, Western Connecticut State University, Danbury, CT 06810

${ }^{13}$ Department of Astronomy, Ohio State University, Columbus, OH 43210 


\begin{abstract}
We present a deep, $42.79 \mathrm{hr}$ image of the nearby, edge-on galaxy NGC 4565 in the Beijing-Arizona-Taipei-Connecticut (BATC) 6660^ band using the large-format CCD system on the $0.6 \mathrm{~m}$ Schmidt telescope at the Xinglong Station of the National Astronomical Observatories of China (NAOC). Following the procedures previously developed by our team for the analysis of deep images of galaxies (Zheng et al.), we obtain a final image that is calibrated to an accuracy of 0.02 mag in zero point, and for which we can measure galaxy surface brightness to an accuracy of $0.25 \mathrm{mag}$ at a surface brightness at $27.5 \mathrm{mag} \operatorname{arcsec}^{-2}$ at $6660 \AA$, corresponding to a distance of $22 \mathrm{kpc}$ from the center of the disk. The integrated magnitude of NGC 4565 in our filter is $m_{6660}=8.99$ (= R magnitude of 9.1) to a surface brightness of $28 \mathrm{mag} \operatorname{arcsec}^{-2}$. We analyze the faint outer parts of this galaxy using a two-dimensional model comprised of three components: an exponential thin disk, an exponential thick disk, and a power-law halo. Combined with a need to provide a cut-off radius for the disk, a total of 12 parameters are included in our model. We determine the best values of our model parameters via 10,000 random initial values, 3,700 of which converge to final values. We then plot the $\chi^{2}$ for each converged fit versus parameter value for each of the 12 parameters. The thin disk and thick disk parameters we determine here are consistent with those of previous studies of this galaxy. However, our very deep image permits a better determination of the power law fit to the halo, constraining this power law to be between $r^{-3.2}$ and $r^{-4.0}$, with a best fit value of $r^{-3.88}$. We find the axis ratio of the halo to be 0.44 and its core radius to be $14.4 \mathrm{kpc}$ (for an adopted distance of $14.5 \mathrm{Mpc}$ ). We also agree with others that the bulge of NGC 4565 is fit well by an exponential luminosity distribution with scale height similar to that found for the thin disk.
\end{abstract}

Subject headings: galaxies: individual (NGC 4565) — galaxies: photometry — galaxies: structure - galaxies: halo - galaxies: bulge 


\section{INTRODUCTION}

In this paper we continue our investigations into the faint surface brightness distributions of edge-on galaxies using data obtained as part of the Beijing-Arizona-Taipei-Connecticut (BATC) Multi-Color Survey of Sky (cf. Fan et al. 1996, Yan et al. 2000). In previous papers detailing our investigations of NGC 5907 (Shang et al. 1998; Zheng et al. 1999), we showed that this galaxy does not have a luminous halo, counter to what was previously suggested (Sackett et al. 1994; Morrison, Boroson, \& Harding 1994). Rather, our images showed a faint ring around NGC 5907, likely the result of the tidal disruption of a dwarf galaxy.

The existence of very faint surface brightness features around edge-on spiral galaxies is further investigated here with BATC observations of the well-known galaxy NGC 4565. As opposed to NGC 5907, NGC 4565 is at high Galactic latitude $\left(86.44^{\circ}\right)$, implying that its halo should be less contaminated by bright Galactic stars than are our observations of NGC 5907. NGC 4565 is classified as Sb de Vaucouleurs et al. 1991). We place it at a distance of $14.5 \mathrm{Mpc}$, based on its distance in the Mark III catalog (1043 km $\mathrm{s}^{-1}$; Willick et al. 1996) and a Hubble constant of $72 \mathrm{~km} \mathrm{~s}^{-1} \mathrm{Mpc}^{-1}$ (Freedman et al. 2001). It is known to have a Seyfert nucleus (Ho et al. 1997), and has been much studied in the past in terms of optical surface photometry (Jensen \& Thuan 1982, hereafter JT; van der Kruit 1979; van der Kruit \& Searle 1981, hereafter KS; Kormendy \& Bruzual 1978; Hamabe et al. 1980; Näslund \& Jörsäter 1997, hereafter NJ; Dettmar \& Wielebinski 1986). Most of those earlier studies were based on photographic data. The V-band data of NJ used a CCD with a relatively small field of view, making it difficult for them to accurately determine sky

background levels. Infrared J, H and K imaging by Rice (1996) completes the existing photometric imaging data on this galaxy.

Our observations and the details of our reduction of the data we have obtained for NGC 4565 are given in Section 2. The measurement of the luminosity profiles and error analysis are presented in Section 3. Section 4 gives the results of model fitting, comparison and possible systematic effects from PSF and disk inclination. The last section summarizes the main results of this paper.

\section{OBSERVATIONS AND DATA REDUCTION}

\subsection{Observations}

Observations of NGC 4565 were obtained with the $60 / 90 \mathrm{~cm}$ Schmidt telescope at the Xinglong Station of the National Astronomy Observatories of China (NOAC), using a thick Ford $2048 \times 2048$ CCD with 15 $\mu \mathrm{m}$ pixels at the $\mathrm{f} / 3$ prime focus. The field of view of this CCD is $58^{\prime} \times 58^{\prime}$ and the scale is $1.7^{\prime \prime} /$ pixel. With the nearly one degree field of view, there is sufficient sky in a single frame such that objects with visual sizes less than $30^{\prime}$ can have their sky background determined accurately. The Lick data-taking 
system is employed and all the CCD images are overscan-subtracted (i.e., initially bias-subtracted) during the readout time (Zheng et al. 1999).

The filter used for the observations reported here is the BATC filter with central wavelength of $6660 \AA$ and bandwidth of $480 \AA$ (Fan et al. 1996 and Yan et al. 2000). This filter is a good compromise between getting as far to the red as possible, and avoiding many bright sky emission lines with the broadest possible filter. Indeed, all of the BATC filters are designed to avoid contamination by emission lines from the night-sky (cf. Fan et al. 1996).

We obtained 190 images of NGC 4565 from 1995 to 1997, of which we deemed 150 images taken in 22 runs (Table 1) as suitable for analysis. Most of these images are of exposure times 900 to $1200 \mathrm{sec}$. In a given night all exposures were dithered randomly at a level of $\sim 10$ pixels to facilitate removal of cosmic rays and CCD defects during the data reduction. The net effect of such dithering is to reduce the full exposure field of view to less than the full field of the CCD. All images used in this analysis have FWHM seeing between 1.7 to 2.6 pixels, or $2.9^{\prime \prime}$ to $4.4^{\prime \prime}$, somewhat larger than the typical seeing at the Xinglong observing station. During the observations the gain of the CCD system was adjusted several times (for various reasons owing to having the CCD system work well), resulting in gains of $3.7 \mathrm{e}^{-} / \mathrm{adu}$ in $1995,4.1$ $\mathrm{e}^{-} / \mathrm{adu}$ in 1996 , and $3.3 \mathrm{e}^{-} / \mathrm{adu}$ in 1997. Readout noise was constant $\left(12 \mathrm{e}^{-}\right)$during the three years.

\subsection{Bias and dark}

The mean value of overscan-subtracted bias was stable within any one month period of time. 10 bias frames ( 5 at the start of the night, 5 at the end) are taken daily for the BATC program. As we can track the stability of the bias during the year, we are able to average from 200 to 300 individual bias frames for each night of observation, which removes any stable structure in the bias frame (any variable level is removed via the overscan subtraction). It is this average of many bias frames that is subtracted from the images of a given night. The CCD dark count has been constantly monitored throughout the BATC survey, and has always been found to be stable and rather free of gradients. As the average dark count/pixel is $3 \mathrm{e}^{-} / \mathrm{hour}$ the dark count level in a 42.8 hour exposure is $128 \mathrm{e}^{-} /$pixel, or $0.04 \%$ of the sky level in this combined image and the spatial variation of dark is even smaller; i.e., of negligible importance. This constant dark count value was scaled to exposure time for each image, then subtracted.

\subsection{Flat Field}

As detailed in our previous papers (cf. Fan et al. 1996; Zheng et al. 1999), the BATC program has developed the means by which we can use dome flats to obtain accurate, high signal-to-noise (S/N), flat fields for flat-fielding sky images. Briefly, the Xinglong Schmidt telescope is equipped with a UV-transparent 
Table 1: Brief observation log of NGC 4565

\begin{tabular}{|c|c|c|c|}
\hline Date & $\mathrm{N}^{\mathrm{a}}$ & Exp.(sec. $)^{b}$ & $\operatorname{Gain}\left(\mathrm{e}^{-} / \mathrm{adu}\right)$ \\
\hline $95 / 01 / 28$ & 1 & 1800 & 3.7 \\
\hline $95 / 03 / 03$ & 8 & 9600 & 3.7 \\
\hline $95 / 03 / 04$ & 2 & 1800 & 3.7 \\
\hline $95 / 05 / 05$ & 2 & 2400 & 3.7 \\
\hline $95 / 05 / 06$ & 7 & 6623 & 3.7 \\
\hline $95 / 05 / 24$ & 4 & 3720 & 3.7 \\
\hline $96 / 01 / 21$ & 4 & 4800 & 4.1 \\
\hline $96 / 02 / 17$ & 17 & 15300 & 4.1 \\
\hline $96 / 02 / 18$ & 20 & 18000 & 4.1 \\
\hline $96 / 02 / 19$ & 20 & 18000 & 4.1 \\
\hline $96 / 02 / 20$ & 16 & 14400 & 4.1 \\
\hline $96 / 02 / 21$ & 4 & 3600 & 4.1 \\
\hline $97 / 03 / 15$ & 4 & 4800 & 3.3 \\
\hline $97 / 03 / 29$ & 3 & 3600 & 3.3 \\
\hline $97 / 03 / 30$ & 1 & 1200 & 3.3 \\
\hline $97 / 04 / 04$ & 4 & 4800 & 3.3 \\
\hline $97 / 04 / 05$ & 6 & 7200 & 3.3 \\
\hline $97 / 04 / 08$ & 5 & 6000 & 3.3 \\
\hline $97 / 04 / 09$ & 7 & 8400 & 3.3 \\
\hline $97 / 04 / 10$ & 5 & 6000 & 3.3 \\
\hline $97 / 04 / 11$ & 6 & 7200 & 3.3 \\
\hline $97 / 05 / 28$ & 4 & 4800 & 3.3 \\
\hline Total & 150 & 154043 & \\
\hline
\end{tabular}


plastic diffuser plate that can be firmly placed directly in front of the Schmidt corrector. The diffuser

provides randomly scattered light to the corrector, reproducing the flux from a uniform sky. Such a method is necessary to accurately flat field our images, as Wild (1997) points out that over a one degree scale, no part of the sky is really flat. Additionally, this fact is attested to by the many BATC images our survey has obtained. We note, however, that it is only with narrow or intermediate-band filters that this diffuser on a Schmidt telescope can produce reliable flat fields. Otherwise, the dome flat field can introduce second-order color terms to broad band observations that must be removed using direct sky images (Fan et al. 1996).

We take twelve dome flats each day, each with exposure times of 150 seconds. This length of exposure reduces the effect of the finite time for shutter opening and closing, resulting in a spurious gradient of less than $0.013 \%$ (cf. Sec. 3.3). All twelve dome flats in one day are combined as the final flat field to correct the frames obtained on the same night. The total count of combined dome flat is about 840,000 electrons per pixel, far higher than that of sky background (about 2,000 electrons per pixel) in a single exposure frame.

\subsection{Image Combination}

Air mass corrections have to be done for our images on a pixel-by-pixel basis, as there exists close to a $1 \%$ gradient in air mass correction over one square degree, even at an altitude of 60 degrees. To take into account the different sets of data (gain differences, dithering, seeing differences, etc.), we combine these images in two steps. First we separate the image frames into 11 groups. Each group includes the frames observed in the same state of instrument system and similar observation conditions (e.g., similar seeing). On average, the observations for a given night are in the same group. The availability of hundreds of well-defined point sources (both stars and distant galaxies) in these frames aided the combination of all frames to a common system. This combination accounts for both dithered frames as well as for slight rotations in the CCD chip from year-to-year. To get all combined images to the same effective seeing radius of 2.3 pixels (4"), some of the combined images were convolved with Gaussian functions to add small additional values of seeing. Three sigma rejection was used to remove cosmic-rays, hot-points, bad pixels and Schmidt telescope-related ghost-images. The final image shown in Figure 1 is the result of merging the 11 combined frames, a total of 42.79 hours of observation.

\subsection{Flux calibration}

Photometric calibration was provided by the five nights that were photometric (Mar. 4, 1995, Mar. 6, 1995, Jan. 6, 1997, Jan. 17, 1997, and Apr. 16, 1997). Following the now-standard BATC photometric calibration (cf. Zhou et al. 2001; Yan et al. 2000), four Oke \& Gunn (1983) standard stars (HD 19445, HD 
84937, $\mathrm{BD}+26^{\circ} 2606$ and $\left.\mathrm{BD}+17^{\circ} 4708\right)$ are used as BATC calibration stars. The calibrations of five nights agree quite well, yielding a zero point accuracy of $0.02 \mathrm{mag}$ as determined from 48 bright stars over the five photometric nights. This zero point yields a sky background in the $6660 \AA$ intermediate-band BATC filter of $20.72 \mathrm{mag} \operatorname{arcsec}^{-2}$, with a corresponding magnitude of $20.30 \pm 0.02 \mathrm{mag}$ for 1 electron second $^{-1}$ in the 42.79 hour image. The sky background is 0.54 mag brighter than it was for our NGC 5907 observations with the same filter (cf. Zheng et al. 1999), likely owing to a combination of most of the images being taken closer to solar maximum (1996-1997) than were the NGC 5907 images, plus an increase of sky brightness over the last few years at the Xinglong observing station (Liu 2001).

\section{MEASUREMENT OF PROFILES AND ERROR ESTIMATE}

Accurate determination of sky background level is a key point for accurate, deep surface photometry. Small variations in sky background, either due to undulations in the CCD sensitivity, or star halos, can affect the measurement of very faint surface brightnesses in galaxies. These issues have been investigated in detail for our data-taking system in our previous investigation of NGC 5907 (Zheng et al.). Here we employ the same methodology as Zheng et al. in obtaining as accurate a fit to the sky background as our data allow.

We first trim our final image to a size of $1701 \times 1701$ pixels $\left(48.2^{\prime} \times 48.2^{\prime}\right)$ centered on NGC 4565 (Figure 1). The small size, relative to our full CCD, is due to the dithering of many images. The average sky level in the final image is close to $302,500 \mathrm{e}^{-} /$pixel, which would nominally yield a statistical error of $550 \mathrm{e}^{-} /$pixel. However, in combining the images the data are effectively smoothed in to an area of somewhat less than $4 \mathrm{pixel}^{2}$, yielding a statistical error of $320 \mathrm{e}^{-} /$pixel for sky in the combined image. In neither case, however, does this statistical error reflect the true error in sky background value, as the true error is convolved with other large-scale sources of variation on the CCD (cf. disucssion below).

\subsection{Star and galaxy masking}

The software SExtractor (Bertin and Arnouts 1996) was used to find all objects in our final image with peak flux higher than 3 sigma above the sky background, as well as to separate stars from some of the fainter galaxies in this field. Following Zheng et al., we also classified all stellar-appearing objects into four types: point sources (stars and faint galaxies) whose wings extend less than 25 pixels at a mask level of 40 $\mathrm{e}^{-}$(about $10 \%$ the sky variation), bright stars (wing radii $>25$ pixels), saturated stars (whose peaks are

Fig. 1.- The full BATC $6660 \AA$ final image of NGC 4565 , comprised of 42.79 hours of observation. The field of view is $1701 \times 1701$ pixels, or $48.2^{\prime} \times 48.2^{\prime}$ in size. 
higher than $10^{7} \mathrm{e}^{-}$) and resolved galaxies. We find more than 12,000 objects in the trimmed image, similar to what we found for the NGC 5907 long exposure images.

To remove as much of the wings of the bright stars as possible, we construct an average PSF from the PSFs of nearly 100 relatively isolated bright stars. In so doing, we can reduce the size needed to mask bright stars by first removing the fainter parts of their PSFs, which are fit using the average PSF. Unfortunately, at the very faint light levels to which our data are sensitive $\left(\sim 29\right.$ mag $\left.\operatorname{arcsec}^{-2}\right)$, we find nearly 10,000 faint objects (almost all distant galaxies at this high Galactic latitude; cf. Odewahn et al. 1996), which limits the radius of our accurate PSF to 25 pixels.

The PSFs of the myriad of faint objects are well fit by this average PSF within a radius of 25 pixels. The fact that we have to restrict the PSF to a radius of 25 pixels is unfortunate for, as luck will have it, too many of the bright stars in this image seem to be near this galaxy (cf. Fig. 1). As shown in Zheng et al., residual light from bright stars affects the measurement of very faint light levels around galaxies. On the other hand, choosing very large masks is not an option, as we find that if we masked out the full PSF of the saturated stars near NGC 4565, we would be left with little of the galaxy to measure.

As a compromise, we first assume that the outer wings of bright and saturated stars are centersymmetric, then later deal with the known asymmetries in the Schmidt PSF. We apply median filters to construct a one-dimensional PSF, whose wings can extend to as far as 300 pixels (510 arcsec) in radius. From this symmetric PSF we construct a two-dimensional PSF and separately subtract it from all the bright and saturated stars. We subtract the wings of the PSF from saturated stars manually to find the best scale factor for each star. The final masks for the saturated stars are designed to be larger than the residual asymmetrical ripples seen in the subtracted PSFs (cf. Zheng et al. 1999). Also sets of small masks are applied to mask the spikes in the Schmidt PSF. Background galaxies and faint stars are masked entirely, with the radius of the masks checked by eye. NGC 4565 itself was masked using three circles: one with a radius of 350 pixels, and two with radii of 250 pixels, The final masked image is shown as Figure 2.

\subsection{Sky background fitting}

The remaining sky background comprises $27.3 \%$ of the area of the final masked image. Before sky fitting, a median filter of $15 \times 15$ pixels is convolved with the masked image using only unmasked pixels. This was done to increase the fraction of area with sky background, as most of masked regions with radii less than 10 pixels are replaced by surrounding background. After such median filtering, the effective sky

Fig. 2. - This is the full mask applied to the $6660 \AA$ final image of NGC 4565, blocking out all point sources as well as a large region about the galaxy itself. 
background increases to $52.8 \%$ of the whole image.

We use the method of Zheng et al. to fit the sky background. Briefly, because of the large masked central region of object galaxies, one-dimensional row or column fitting with functions of high order will introduce uncontrolled fluctuations in the fitted sky. To avoid this, a one order Spline3 fit was applied in one-dimension, both row-by-row and column-by-column separately (cf. Zheng et al.), using only those unmasked points with backgrounds $<2.5 \sigma$ of the average. We then average the row-fitted and column-fitted images and smoothed the average with a circular-gaussian filter of $\sigma=30$ pixels. This smoothed fit is then adopted as the sky background and subtracted from the final image.

As a test, we also use one-dimensional, low-order Legendre polynomials in place of the Spline3 fits. The difference between the two sky images in the central region around NGC 4565 is less than $40 \mathrm{e}^{-}$pixel $^{-1}$ (out of a sky background of 302,500 $\mathrm{e}^{-}$pixel $^{-1}$ ). Hence, determination of the sky background is not significantly affected by the spline-fitting function used. To estimate the systematic error of sky-fitting, we again mask the background subtracted image and separate the image into 400 adjoining regions of $85 \times 85$ pixels. The standard variation of mean values of these $85 \times 85$ boxes is $115 \mathrm{e}^{-}$pixel $^{-1}$. This is regarded as the best estimate of the added error introduced by our sky-fitting procedure. Figure 3 shows the plots of four slices of sky background-subtracted image, which shows the accuracy of the method (cf. Figures 7 and 8 in Zheng et al.).

\subsection{Error estimate}

There are two types of errors that can affect our results. One type is random and yields a Poisson distribution that can be suppressed by increasing the number of sampled points. The another type is systematic in nature, and is usually independent of sample size. In the following, we will discuss the error sources individually.

\section{Readout noise}

Readout noise is random noise which is introduced in the process of data readout. For our CCD, the value is $12 \mathrm{e}^{-}$pixel $^{-1}$ per frame. Because the combined image is made of 150 frames, the final noise is 147.0 $\mathrm{e}^{-}$pixel $^{-1}$. The bins used for the analysis of the light distribution in the halo of NGC 4565 vary in size from 24 pixels (a box of $8 \times 3$ pixels) to 1750 pixels (a box of $35 \times 50$ pixels). Hence, when averaged in this way, the readout noise is $30.0 \mathrm{e}^{-}$for the smallest bins, and $3.5 \mathrm{e}^{-}$for the largest bins.

\section{Sky background photon noise}

As discussed above, the merging process to produce the final image effectively smooths the data, such that the flux in each pixel is an average of nearly 4 pixel $^{2}$ around it. Hence, in the final image, the photon 


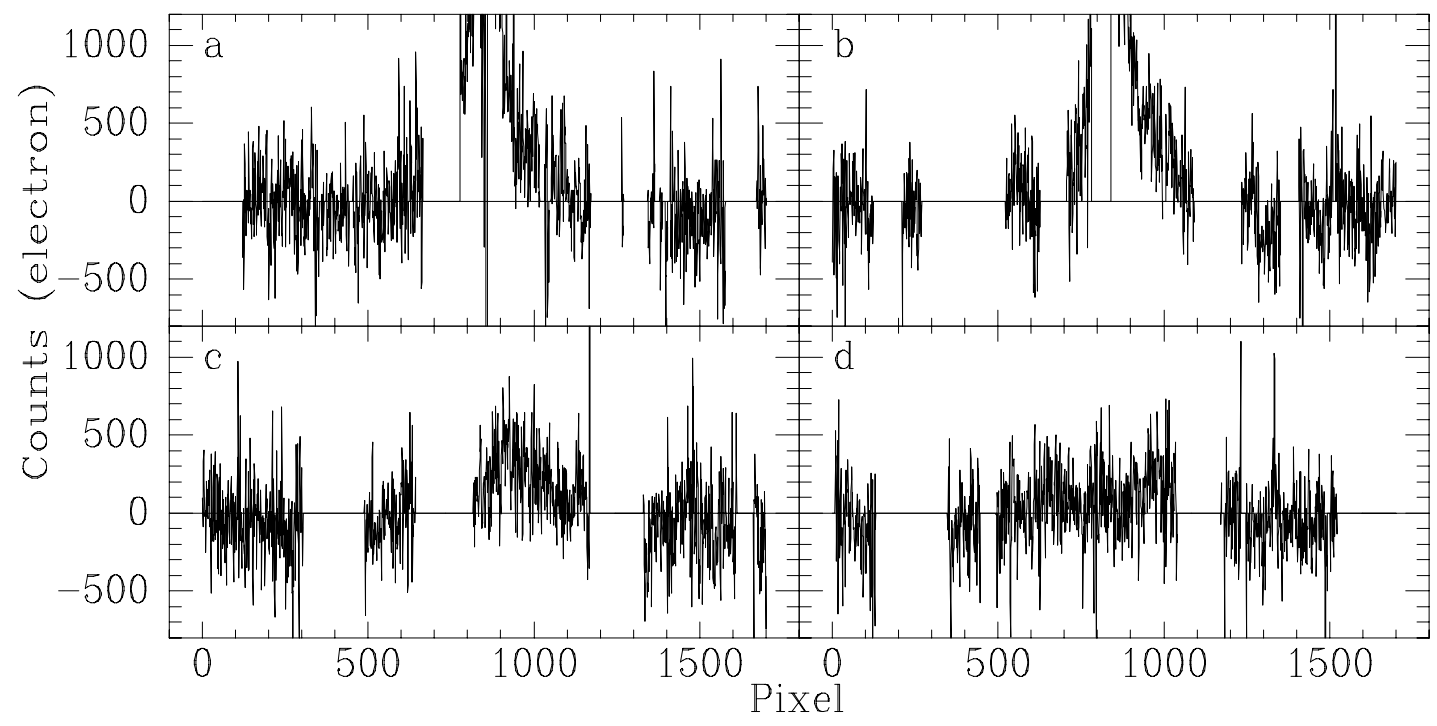

Fig. 3.- Four slices of the background-subtracted $6660 \AA$ image to show the accuracy of the sky background fit. Each slice is averaged over 35 lines of the image, and only non-masked pixels are used. If all 35 points are masked, a zero value is assigned. The straight lines indicate the zero level. a) 8 arc-minute SE of the minor axis. b) 8 arc-minute NW of the minor axis. c) 8 arc-minute SW of the major axis. d) 8 arc-minute $\mathrm{NE}$ of the major axis. The large scale variation of background subtraction is small. The disk of NGC 4565 is seen in the centers of slices (a) and (b). The wings of subtracted stars are also evident in these slices. 
error per pixel is $320 \mathrm{e}^{-}$, as opposed to the $550 \mathrm{e}^{-}$one might otherwise expect from a mean sky background of $302,500 \mathrm{e}^{-}$pixel $^{-1}$. Using this value, we estimate the photon sky noise to be $15 \mathrm{e}^{-}$for a region $35 \times 50$ pixels in size (our largest bin size), and $130.6 \mathrm{e}^{-}$for a region $8 \times 3$ pixels in size (our smallest bin size).

Dark current noise

The dark current of our combined image of 42.79 hour is $128.3 \mathrm{e}^{-}$pixel $^{-1}$. This introduces random noise of $11.3 \mathrm{e}^{-}$pixel $^{-1}$. For the smallest and largest bins, this value reduces to $2.3 \mathrm{e}^{-}$and $0.27 \mathrm{e}^{-}$separately.

\section{Bias subtraction noise}

Since the readout noise is $12 \mathrm{e}^{-}$pixel $^{-1}$ and 200 to 300 bias frames are used to form final average bias, the error from bias subtraction is about $0.8 \mathrm{e}^{-}$pixel $^{-1}$, yielding $0.02 \mathrm{e}^{-}$pixel $^{-1}$ for the region of $35 \times 50$ pixels and $0.17 \mathrm{e}^{-}$for the smallest bins used here.

\section{Flat field: Random and Systematic Noise}

The averaged nightly flat used here is comprised of 12 dome-flats with total counts about 840,000 $\mathrm{e}^{-} /$pixel, yielding a photon noise close to $0.1 \%$, or $330.1 \mathrm{e}^{-}$pixel $^{-1}$ equivalent sky background counts. Given that that final image includes 150 shifted, averaged flats the error per pixel is reduced to $0.009 \%$. This part of the flat field error is random in nature and translates to a formal error of $0.0002 \%$ for the largest sample bins used here, to $0.0018 \%$ for the smallest bins.

The stability of the large-scale flat field was checked by comparing the dome-flats from adjacent nights. The average results of these tests indicate an error of $0.03 \%$, or close to $90 \mathrm{e}^{-}$pixel $^{-1}$ for our measured sky background. In our CCD system, the opening and closing time of shutter is 20 milliseconds and the exposure time of each dome-flat is 150 seconds. So there exists a $0.013 \%$ gradient due to finite shutter speed in the each dome-flat frame. Combining these two sets of errors, we estimate the error from systematic variations in the large-scale flat-field is $0.033 \%$, equivalent to $100 \mathrm{e}^{-}$pixel $^{-1}$ of sky background.

\section{Intrinsic variation in galaxy brightness}

Following MBH (Morrison et al. 1994) and Zheng et al., we can calculate the random error due to intrinsic variation in the surface brightness of the galaxy. In the terminology introduced by Tonry and Schneider (1988), $\mathrm{m} 1$ for our image is $20.30 \mathrm{mag}$, the exposure time is $154,043 \mathrm{sec}$, the distance is $14.5 \mathrm{Mpc}$ and $\overline{\mathrm{M}}_{6660}$ is adopted as 0 (Zheng et al. 1999). In the case of $300 \mathrm{e}^{-}$pixel $^{-1}$ from the galaxy (equal to a surface brightness of $28.23 \mathrm{mag} \operatorname{arcsec}^{-2}$ ), this error is $53.8 \mathrm{e}^{-}$pixel $^{-1}$, or an error of 18 translates to an average error of $1.29 \mathrm{e}^{-}$for the largest bins used here, to $10.98 \mathrm{e}^{-}$for the smallest bins.

\section{Background subtraction}

There are two types of background subtraction errors, both dominated by systematic effects. One is the accuracy of the sky background subtraction, for which we adopt the value of $115 \mathrm{e}^{-}$obtained above. The 
second comes from imperfect star subtraction, especially for saturated stars. For this we adopt an average error of $100 \mathrm{e}^{-} /$pixel by checking the regions in which stars are subtracted. We acknowledge this error can be significantly higher for selected stars, especially for the saturated stars which are so close to NGC 4565 . For the true errors involved in star subtraction, we must look to the consistency of the luminosity profiles at faint surface brightness levels.

\subsection{The Total Error Budget}

All the sources of errors discussed are listed in Table 2. We assume that the mean count per pixel from the object galaxy is $300 \mathrm{e}^{-}$(i.e., in its faint halo), a sky level of $302,500 \mathrm{e}^{-}$and bin sizes of $35 \times 50$ pixels and $8 \times 3$ pixels for surface photometry. This leads to a final error of close to $400 \mathrm{e}^{-} \mathrm{pixel}^{-1}$. Errors for each bin are calulated based on the number of non-masked pixels in that bin, ratioed to the error expected for all pixels in that bin having data.

As photon noise in the sky is the dominant source of error in our data, we give the expected error per bin for both the smallest bin we use for $R$-profiles $(8 \times 3$ pixels $)$ and the largest bin we use for $z$-profiles $\left(35 \times 50\right.$ pixels). The error for the faintest part of NGC 4565 is close to $183 \mathrm{e}^{-}$pixel $^{-1}$, or $63 \mathrm{e}^{-} \operatorname{arcsec}^{-2}$. That is, the relative error of measured flux at $28.77 \mathrm{mag} \operatorname{arcsec}^{-2}$ is $100 \%$, leading to an error bar of 0.75 mag $\operatorname{arcsec}^{-2}$. This also corresponds to an error of $0.25 \mathrm{mag} \operatorname{arcsec}^{-2}$ at a surface brightness of $27.5 \mathrm{mag}$ $\operatorname{arcsec}^{-2}$. In fact, the error is a little higher than this, because there exist masked regions in the measured boxes. The main sources of error are from large-scale variation of flat-field, sky fitting and residuals from star subtraction.

\section{ANALYSIS AND RESULTS}

\subsection{Luminosity profiles}

As others have done (cf. NJ), we use a contour map to determine the position angle of the major axis. Then the sky-subtracted image is rotated to that angle to put the major axis of the object galaxy along the $\mathrm{x}$-axis direction. At the same time, all circular masks but the three masks used for the object galaxy were transformed to the new coordinate system and imposed on the rotated image (Figure 4).

For purposes of analysis, we measure the luminosity profiles of NGC 4565 in two orthogonal directions.

Fig. 4.- This is the image that results from sky-subtracting a two-dimensional background from the final image, with the final point source masks still in place. We have rotated the image so that the major axis of the galaxy lies along the x-axis. Here we only present the central $700 \times 700$ pixels region of the image. 
One direction is parallel to the galaxy minor axis (the " $z$ " direction), the other is parallel to the major axis (the " $R$ " direction). We sample the $z$ direction in discrete lengths (parallel to the major axis) of 35 pixels ( 1 arcmin) on both sides of the major axis. We employ bins of varying width perpendicular to the major axis (i.e., along the $z$ direction): from 1 pixel when $z=0$ (i.e., on the major axis) to 50 pixels for the largest $z$ distances $\left(\sim 7^{\prime}\right)$. This methodology yields bin sizes that vary from $35 \times 1$ pixels to $35 \times 50$ pixels. Only unmasked pixels are used, and the flux cited is the median value for that bin. The measured $z$ and $R$ profiles are shown in Figure 5. Here we adopt a distance of $14.5 \mathrm{Mpc}$, with $1^{\prime \prime}=0.0703 \mathrm{kpc}$.

We see that the four $z$-profiles in each plot (i.e., all four quadrants of the galaxy) agree reasonably well down to surface brightness of $28 \mathrm{mag} \operatorname{arcsec}^{-2}$, or a level of $0.12 \%$ of sky) within a radius of 6 arcmin. Greater than that distance, the well-known warp in the disk of this galaxy (SE to NW) (cf. Sancisi 1976; van der Kruit 1979; Näslund \& Jörsäter 1997; see below) begins to break down the symmetry. The $z$-profiles are tabulated in Table 3 (full table in electronic form).

The $R$ series of profiles are parallel to the major axis with $z$ distance: $0^{\prime \prime}, 10^{\prime \prime}, 20^{\prime \prime}, 30^{\prime \prime}, 40^{\prime \prime}, 60^{\prime \prime}, 80^{\prime \prime}, 100^{\prime \prime}$ and $120^{\prime \prime}$ respectively. Since the $z$-profiles decreases quickly at small $z$ and relatively slow at larger $z$, the sizes of rectangles are changed from $8 \times 3$ pixels at $z=0^{\prime \prime}$ to $8 \times 11$ pixels at $z=120^{\prime \prime}$. The measured $R$

profiles are also in Figure 5. The asymmetries of the NW and SE parts are also shown in $R$-plots. The data are listed in Table 4.

\subsection{Warp}

Figure 6 shows the central points of the profiles parallel to the minor axis. This was determined by keeping the upper and lower profiles in the best agreement. By plotting these central points as a function of radius in this galaxy, we map out its warp from SE to NW sides. The NW warp was first found in HI (Sancisi 1976) and the optical warp of both sides was discussed by NJ.

The figure shows that the stellar warp starts at $21 \mathrm{kpc}$ from the center of galaxy and is less distorted than that of NGC 5907 (Zheng et al. 1999; Morrison et al. 1994), whose warp starts from $4.1 \mathrm{kpc}$. In fact, the starting points of warp are asymmetric: $21 \mathrm{kpc} \mathrm{NW}$ and $25 \mathrm{kpc}$ SE. This causes the asymmetries in Fig. 5.- The $z$-profiles (upper plots) and $R$-profiles (lower plots) of NGC 4565 from our $6660 \AA$ final image. The four quadrant profiles of the galaxy (except the minor and major axes) are plotted in each sub-plots. The $z$-profiles agree reasonably well down to $28 \mathrm{mag}$ within a radius of 6 arcmin. The obvious asymmetries of the NW and SE parts are shown in both $z$-plots and $R$-plots. The dust lane is indicated by arrows in the first $3 z$-plots. The solid line is our two-disk + halo model. The dash-dot and dashed lines are the thin and the thick disk models respectively, and the dotted line is the power-law halo. All the data are well-fitted by this model. Here one arcsec is $0.0703 \mathrm{kpc}$. 
Table 2: The error estimation

\begin{tabular}{lrrrrrr}
\hline \hline Source of variation & $\mathrm{e}^{-} /$pixel & $\%$ & $35 \times 50 \mathrm{bin}\left(\mathrm{e}^{-}\right)^{a}$ & $\%$ & $8 \times 3$ bin $\left(\mathrm{e}^{-}\right)^{a}$ & $\%$ \\
\hline Readout noise & 147.0 & 0.049 & 3.5 & 0.001 & 30.0 & 0.010 \\
Photon noise of sky & 320.0 & 0.106 & 15.0 & 0.005 & 130.6 & 0.043 \\
Dark & 11.3 & 0.004 & 0.27 & 0.0001 & 2.3 & 0.0008 \\
Bias (formal) & 0.8 & 0.0003 & 0.02 & 0.0000 & 0.17 & 0.0001 \\
Flat field (small-scale/pixel scale) & 26.9 & 0.009 & 0.6 & 0.0002 & 5.1 & 0.0017 \\
Flat field (large-scale/> 100 pixel scale) & 100.0 & 0.033 & 100.0 & 0.033 & 100.0 & 0.033 \\
Surface brightness fluctuations ${ }^{b}$ & 53.8 & 0.018 & 1.29 & 0.0004 & 10.98 & 0.0036 \\
Background subtraction & 115.0 & 0.038 & 115.0 & 0.038 & 115.0 & 0.038 \\
Star subtraction & 100.0 & 0.033 & 100.0 & 0.033 & 100.0 & 0.033 \\
& & & & & & \\
Total & 401.2 & 0.133 & 182.9 & 0.060 & 226.6 & 0.075 \\
\hline
\end{tabular}

$\bar{a}$ The error in surface photometry for bins of $35 \times 50$ or $8 \times 3$ at a count of sky level

${ }^{b}$ Here assuming $300 \mathrm{e}^{-}$pixel $^{-1}$ from the galaxy.

Table 3: Surface brightness of $z$-profiles of NGC 4565 in i( $6660 \AA)$-band

\begin{tabular}{|c|c|c|c|c|c|}
\hline $\begin{array}{l}R \\
(\operatorname{arcmin})\end{array}$ & $\begin{array}{c}z \\
(\operatorname{arcsec})\end{array}$ & $\begin{array}{c}z \\
(\mathrm{kpc})\end{array}$ & $\begin{array}{c}\mathrm{i}(6660 \AA) \\
\left(\mathrm{mag} / \operatorname{arcsec}^{2}\right)\end{array}$ & $\begin{array}{c}\text { High Error } \\
\left(\mathrm{mag} / \operatorname{arcsec}^{2}\right)\end{array}$ & $\begin{array}{c}\text { Low Error } \\
\left(\mathrm{mag} / \operatorname{arcsec}^{2}\right)\end{array}$ \\
\hline \multirow[t]{10}{*}{0.00} & 2.57 & 0.18 & 19.50 & 0.04 & 0.04 \\
\hline & 5.99 & 0.42 & 20.27 & 0.03 & 0.03 \\
\hline & 9.41 & 0.66 & 20.59 & 0.02 & 0.02 \\
\hline & 12.83 & 0.90 & 20.37 & 0.02 & 0.02 \\
\hline & 16.25 & 1.14 & 20.07 & 0.02 & 0.02 \\
\hline & 19.67 & 1.38 & 19.85 & 0.01 & 0.01 \\
\hline & 23.09 & 1.62 & 19.93 & 0.01 & 0.01 \\
\hline & 26.51 & 1.86 & 20.16 & 0.01 & 0.01 \\
\hline & 29.93 & 2.10 & 20.42 & 0.02 & 0.02 \\
\hline & 33.35 & 2.34 & 20.73 & 0.02 & 0.02 \\
\hline
\end{tabular}

Note.- Only a portion of Table 3 listed here. 
$z$-plots and $R$-plots (Figure 5). The amplitude of the warp increases rapidly after it begins, becoming more than $1 \mathrm{kpc}$ from the centrally-defined major axis after a distance of $30 \mathrm{kpc}$. We plan to take further deep images of NGC 4565 to better explore its fainter stellar populations, including those in its warp.

\subsection{Comparison with previous work}

Jensen and Thuan (1982) present the luminosity profile of NGC 4565 in the B and R bands, using photographic data. As our intermediate-band i filter centered at $6660 \AA$ is quite similar to R-band (cf. Fan et al. 1996), it is straightforward to compare with JT's results. Zhou et al. (2001) give the transformation between the BATC i-band and R-band as: $\mathrm{R}=\mathrm{m}_{6660}+0.1048$. The data of JT are then compared to our own as a function of the $z$ direction for six values of $R$, as shown in Figure 7. Here we plot the difference between observed profiles and our fitted profile, as a function of $z$ distance. In general, the JT's data (upper plots) agree with ours (lower plots) within $0.5 \mathrm{mag}$, but the data of JT become systematically fainter than ours at large radii and $z$-distances. Given that the data of JT are photographic, their faint values are more subject to larger systematic problems than ours.

\subsection{The adopted model}

Previous studies have shown that one single disk can not fit the fainter parts of the $z$-profiles of NGC 4565 reasonably (Jensen \& Thuan 1982; Shaw \& Gilmore 1989; Näslund \& Jörsäter 1997). Rather, for the $z$ profiles we adopt a three-component model: thin disk (seen at inclination $i$ ), thick disk (cf. Burstein 1979), and halo. The $z$ structure for the thin and thick disks adopted is that of the $\operatorname{sech}^{2}(z)$ model (van der Kruit \& Searle 1981), which has a density distribution of a self-gravitating isothermal sheet in the $z$ direction. Alternate fits proposed by de Grijs \& van der Kruit 1996 (i.e., exponential or $\operatorname{sech}(z) \operatorname{light}$ distributions), while fitting better at small $z$ values, are not as physically motivated as the $\operatorname{sech}^{2}$ model. Moreover, all three models show the similar behavior at large $z$, showing that the different $z$ models do not affect the model of the halo one derives for edge-on galaxies. A standard exponential disk was used in the radial direction $(r)$, well away from the bulge.

The density distribution of both the thin and the thick disks is described by

$$
\rho(r, z)=\rho_{0} e^{-\frac{r}{h_{r}}} \operatorname{sech}^{2}\left(\frac{z}{z_{0}}\right),
$$

with $z_{0}$ and $h_{r}$ as the disk scale height and scale length and $z_{0}$ equal to twice the scale height $h_{z}$ of the exponential model. As galaxy disks do not go to infinity in either the $z$ or the $r$ directions (cf. Figure 4), we introduce a cutoff in the $r$-distribution at $r_{\max }$, beyond which for $r>r_{\max }, \rho(r, z)=0$. For an edge-on 
Table 4: Surface brightness of $R$-profiles of NGC 4565 in the i( $6660 \AA)$-band

\begin{tabular}{lccccc}
\hline \hline$z$ & $R$ & $R$ & $\begin{array}{c}\mathrm{i}(6660 \AA) \\
(\operatorname{arcsec})\end{array}$ & $\begin{array}{c}\text { High Error } \\
(\operatorname{arcsec})\end{array}$ & $\begin{array}{c}\text { Low Error } \\
(\mathrm{kpc})\end{array}$ \\
\hline 0.00 & 0.00 & 0.00 & 17.55 & 0.10 & 0.11 \\
& 10.26 & 0.72 & 18.45 & 0.09 & 0.09 \\
& 20.52 & 1.44 & 18.85 & 0.06 & 0.06 \\
& 30.78 & 2.16 & 19.23 & 0.07 & 0.08 \\
& 41.04 & 2.89 & 19.57 & 0.07 & 0.07 \\
& 51.30 & 3.61 & 19.74 & 0.06 & 0.06 \\
& 61.56 & 4.33 & 20.01 & 0.07 & 0.07 \\
71.82 & 5.05 & 20.22 & 0.06 & 0.07 \\
& 82.08 & 5.77 & 20.53 & 0.02 & 0.02 \\
92.34 & 6.49 & 20.53 & 0.02 & 0.03 \\
\hline
\end{tabular}

Note.- Only a portion of Table 4 listed here.

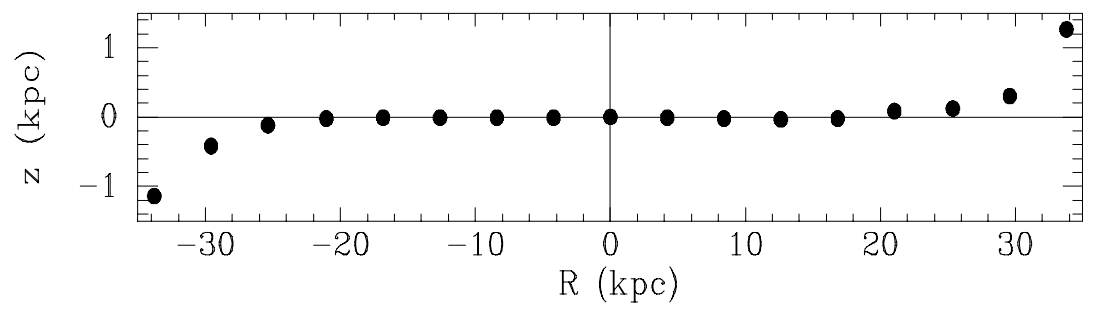

Fig. 6. - The stellar warp of NGC 4565 measured in $6660 \AA$ band. The x-axis is along the major-axis of galaxy. The left is to the south-east (SE) and the right is to the north-west (NW). The warp can be seen on both sides of this galaxy, but starting at different distances on either side. 

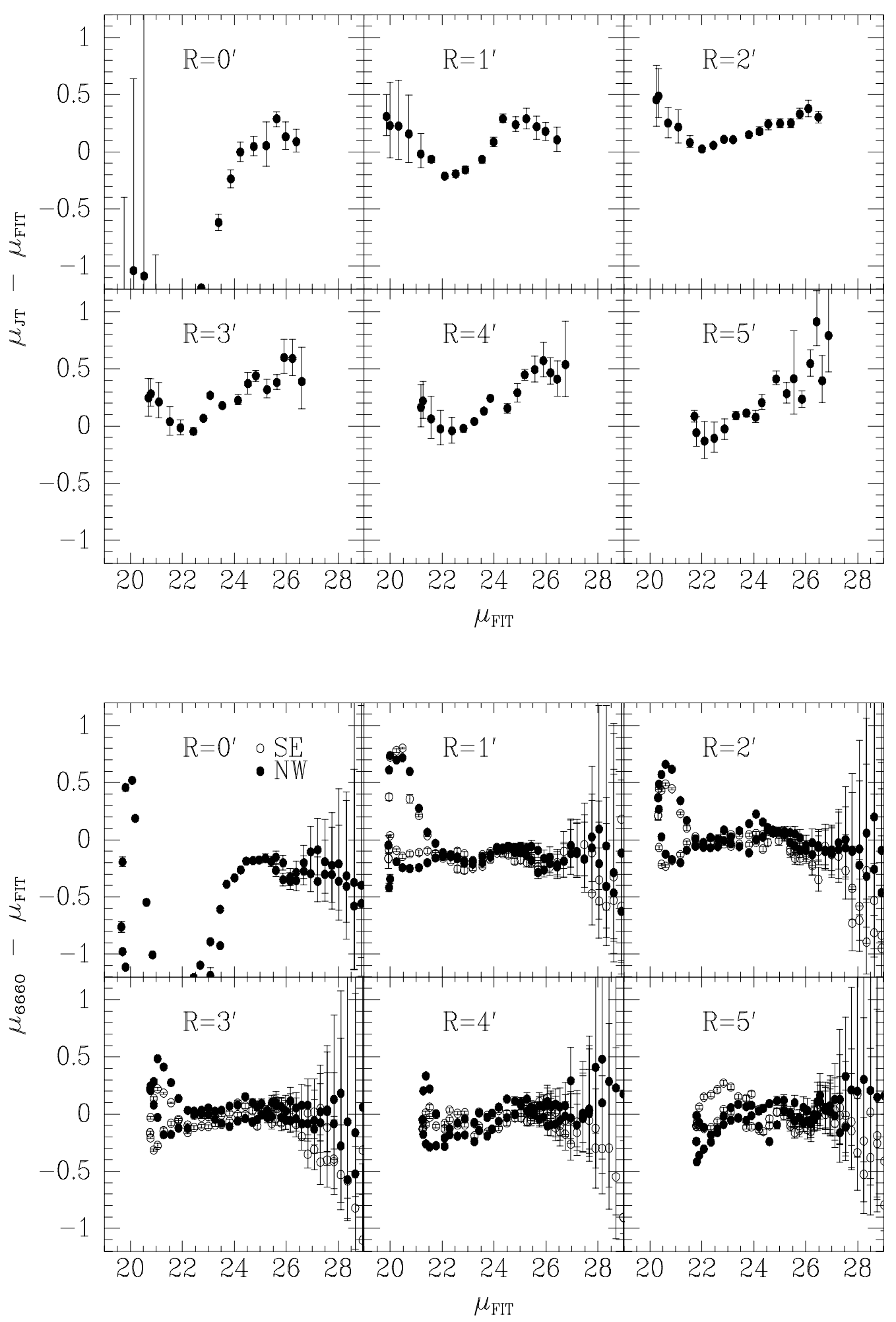

Fig. 7.- Comparison of our $6660 \AA$ data with those of JT. Differences between observed profiles (JT, upper plots; and ours, lower plots) and our fitted profiles are plotted against model surface brightness. In the lower plots, the solid circles are data from the NW side of the galaxy and open circles are data from the SE side. 
galaxy, this means

$$
\mu(R, z)=\mu_{0}-2.5 \log 10\left[2 \operatorname{sech}^{2}\left(\frac{z}{z_{0}}\right) \int_{R}^{r_{\max }} \frac{e^{-\frac{r}{h_{r}} r}}{\sqrt{r^{2}-R^{2}}} d r\right] .
$$

Here, $R$ is the projected distance from the center along the major axis.

A power-law halo is introduced as the third component, with its luminosity distribution modeled as in NJ:

$$
\rho(r, z)=\frac{\rho_{0}}{\left[1+\frac{r^{2}+\left(\frac{z}{q}\right)^{2}}{r_{0}^{2}}\right]^{\frac{\gamma}{2}}},
$$

where $r_{0}$ is the core radius, $q$ is the axis ratio and $\gamma$ is the power index. For an edge-on galaxy, this model has the form (where $\Gamma$ is the standard Gamma function):

$$
\mu(R, z)=\mu_{0}-2.5 \log 10\left\{\sqrt{\pi} \frac{\Gamma\left(\frac{\gamma-1}{2}\right)}{\Gamma\left(\frac{\gamma}{2}\right)} r_{0}^{\gamma}\left[r_{0}^{2}+R^{2}+\left(\frac{z}{q}\right)^{2}\right]^{\frac{1-\gamma}{2}}\right\}
$$

\subsection{Profile fitting}

\subsubsection{Data used for fitting}

As its dust lane nearly bisects the major axis of NGC 4565, it is difficult for us to fit all the components of this galaxy (nucleus, bulge, disks and halo) simultaneously. The primary purpose of the present paper is to search for luminous halos in edge-on galaxies. Hence, we concentrate only on the three components whose luminosity distributions affect the halo of this galaxy: thin disk (via projected major axis), thick disk and halo. To avoid the possible effect of nucleus, bulge, dust lane and warps, only the $z$-profiles with $2^{\prime} \leq R \leq 7^{\prime}$ were used in the fit. For $R$-profiles, we rejected the profiles along the dust lane and the points within bulge. Though there exists obvious asymmetries between the NW and SE parts of galaxy, they are treated here the same owing to the fact that large areas in both sides of the galaxy are masked.

A general $\chi^{2}$ method was used to estimate the parameters of the fits. In principle, one should use a weighted fitting scheme (cf. Shaw \& Gilmore 1989) of the form:

$$
\chi^{2}=\sum_{i}\left[w_{i}\left(\mu_{i}-\mu_{f i t}\right)\right]^{2},
$$

with $\mu_{i}$ and $\mu_{f i t}$ as observed and fitted surface brightnesses. In practice, the values assigned to $w_{i}$ depend on what one is trying to fit. Unfortunately, the manner in which we can compensate for fainter surface brightness levels (by using larger sample bins) does not fully compensate for the observational errors in 
the outer regions, which are much larger than those in the inner regions. In addition, by sampling with different bin sizes, we have more measurements nearest the major axis than we do far away from the major axis. Thus, we have more points in our fit for the inside regions with small uncertainties than those for the outside with large uncertainties. Strictly error-weighting the data would bias the fits toward the innermost data. As such, we chose to give equal weight to the bins, which better accounts for the larger number of bins close to the major axis. The fitting algorithm was implemented using the Levenberg-Marquardt technique, with the subroutine used taken from Numerical Recipes (Press et al. 1992).

\subsubsection{Estimates of parameters}

Three parameters must be determined before fitting: the coordinates of the galaxy center $x_{c}$ and $y_{c}$ and the sky background value. The latter component has already been removed from the data to be analyzed. The effect of inclination is neglected here (but considered later), as NGC 4565 is so nearly edge-on. This leaves us 12 parameters to fit, namely: 3 surface brightness $\mu_{01}, \mu_{02}$ and $\mu_{03}$ (with $1=$ thin disk; $2=$ thick disk and $3=$ halo), the scale heights $z_{01}$ and $z_{02}$ of thin and thick disks components in $z$-direction and scale lengths $h_{r 1}$ and $h_{r 2}$ in radial direction, the cutoff radius $r_{\max 1}$ and $r_{\max 2}$ of two kinds of disks, and the core radius $r_{0}$, axis ratio $\mathrm{q}$ and power index $\gamma$ of the halo.

Unfortunately, we have too many parameters for the fitting procedure to converge successfully. As discussed by Fry et al. (1999), for three free parameters, the initial estimates need to be within $20 \%$ of the correct values for the model to converge. At the same time, the existence of many local minima make the final fit strongly dependent on the initial estimate of these parameters.

To find the true minimum and the best fit, we randomly selected 10,000 sets of 12 initial parameters spaced uniformly as to cover the reasonable range covered by these parameters (Table 5). Of these 10,000 tries, we found nearly 3,700 convergences. The $\chi^{2}$ for these 12 parameters from these 3,700 convergences are plotted against the value for finding for those convergences in Figure 8. The minimum is marked as symbol star in each sub-plot.

Table 5: The confined ranges of initial parameters.

\begin{tabular}{lll}
\hline \hline Thin disk & Thick disk & Halo(power-law) \\
\hline$\mu_{0}: 20-23 \mathrm{mag} \mathrm{arcsec}^{-2}$ & $\mu_{0}: 23-27 \mathrm{mag} \mathrm{arcsec}^{-2}$ & $\mu_{0}: 22-27 \mathrm{mag} \operatorname{arcsec}^{-2}$ \\
$z_{0}: 0.6-1.5 \mathrm{kpc}$ & $z_{0}: 1.2-2.9 \mathrm{kpc}$ & $r_{0}: 0-22 \mathrm{kpc}$ \\
$h_{r}: 5.8-11.6 \mathrm{kpc}$ & $h_{r}: 7.2-29.0 \mathrm{kpc}$ & $\gamma: 2-5$ \\
$r_{\max }: 29-38 \mathrm{kpc}$ & $r_{\text {max }}: 32-39 \mathrm{kpc}$ & $q: 0.1-0.9$ \\
\hline
\end{tabular}


The distributions of $\mu_{01}, z_{01}, h_{r 1}$ (i.e., the thin disk component) and $q$ (the axis ratio of the halo) are well determined by our data. The axis ratio of the halo we obtain (0.44) is in good agreement with that of NJ, who show that $q=0.5$ is a better fit than $q=1.0$ for a two-disk-plus-halo model in the V-band.

The parameters of the thick disk and the power-law halo have wide $\chi^{2}$ distributions. The scale height of the thick disk $(2.55 \mathrm{kpc})$ is about twice of that of the thin disk and agrees with the $h_{z}=1 \mathrm{kpc}\left(h_{z}=z_{0} / 2\right)$ given by Burstein 1979 for S0 galaxies. The thick disk scale length is $11.03 \mathrm{kpc}$, larger than that of the thin disk. The luminosity of the thick disk is $20 \%$ of the thin disk. The core radius of the power-law halo is 14.4 kpc, indicating a flat halo. This agrees with the third component of three disk models of NJ and that of Shaw \& Gilmore (1989), in which the scale lengths of their third disks are about 13.9 kpc (at a distance of 14.5 Mpc). The values of $\gamma$ concentrate in the range of 3.2 to 4.0 , which indicates that there does not exist a $r^{-2}$ halo in this galaxy. The best power index is 3.88, between the 3.5 of Milky Way (Zinn 1985) and the 4.0 of M31 (Pritchet \& van den Bergh 1994). The $r_{\max }$ of the thin and the thick disks show several local minimums, owing to data sampling. The cutoffs of the thin and thick disks are about $32 \mathrm{kpc}$ and $37 \mathrm{kpc}$, respectively.

Analyses of correlation between any two parameters show that some of the parameters are correlated, which means some of the parameters can not be determined independently. For example, we find correlation between $z_{01}$ and $z_{02}$ and among $\mu_{03}, r_{0}$ and $\gamma$. The similar $\chi^{2}$ distributions of $\mu_{03}, r_{0}$ and $\gamma$ in Figure 8 also show such a correlation.

The error for each parameter is obtained in the following way. Random values are selected for the observed data such that they obey a normal distribution, with sigmas determined by the known errors in each sampled bin. We then obtain the best-fitted parameters for that set of data. This procedure is repeated three hundred times, giving us 300 separate determinations of the best-fitted value for each parameter. The statistical standard deviation of each parameter from this procedure is adopted as the final error for this parameter.

The best fit parameters and errors are listed in Table 6 . Table 6 also lists the total magnitude in $6660 \AA$-band to a surface brightness of $28 \mathrm{mag} \operatorname{arcsec}^{-2}, \mathrm{~m}_{6660}=8.99$ (or broad-band $\mathrm{R}=9.10$ from the Zhou et al. transformation), which is measured by replacing masked areas around the galaxy by the corrsponding parts of the unmasked galaxy.

Figure 5 presents the best fitting values for the three components compared to our data in both the $z$

Fig. 8. - The values of the $\chi^{2}$ for the 3,700 random fits that converged, versus the value of the parameters at which they converged. The star symbol in each sub-plot is the minimum point adopted as our best-fitting value. The values of $\gamma$ concentrate in the range of 3.2 and 4.0, ruling out the existence of luminous halo in NGC 4565. 
and $R$ directions. The fits are quite good in the range of $R=1^{\prime}$ to $R=8^{\prime}$ for the $z$-profiles and in the range of $z=30$ " to 120 " for $R$-profiles. As expected, the model deviates significantly from the data in the region of the bulge (not-fitted) and where the warp of the disk becomes sizeable.

Table 7 compares the dimensional parameters obtained by this study and to similar parameters determined by other studies of NGC 4565, as magnitude zero points are too dependent on the filter that was used. The thin disk parameters are consistent among the different analyses. Our value of $8.05 \mathrm{kpc}$ for the thin disk exponential scale length and $1.17 \mathrm{kpc}$ for its scale height are close to those obtained by R96, KS, NJ and SG, scaled to our adopted galaxy distance. The scale height and scale length of our fits to the thick disk agree best with the thick disk in SG's two-disk $+\mathrm{R}^{1 / 4}$ halo model. The thick disk components of our model are somewhat larger than the thick disk in the three-disk models, likely owing to the halo being much flatter in the inner region of NGC 4565 than the models for the third disk used by these other studies.

\subsection{Bright Versus Faint Parts of NGC 4565}

As our data go very faint, one worry is that the very faintest parts of the PSFs of the bright stars could interfere with our measurement of the halo of this galaxy (such as we found to be the case in our analysis of NGC 5907; cf. Zheng et al.). As our final image of NGC 4565 did not permit us to fully test the faintest parts of the PSF in a reliable manner, we separately observed a set of bright stars with the same CCD parameters. From those observations we construct a new PSF that can extend to 1700 arcsec, compared to the extension of only 510 arcsec permitted by our final NGC 4565 image. (In this we note that even 510 arcsec is still farther than the radii considered by either Morrison et al.(1994) for NGC 5907 or Fry et al.(1999) for NGC 4244). The agreement between the PSF we obtain from our NGC 4565 image and that obtained from the special bright star images is excellent where they overlap (i.e., interior to 510 arcsec).

There are two effects that can, in principle, affect our halo results. One is scattered light from the nucleus and bulge. The other is the scattered light from the brighter parts of the galaxy as a whole. To test the first effect, the nucleus and bulge is separated from the other parts of galaxy by subtracting our best fitting model from the final image (cf. Figure 11). We then convolve the resulting nucleus and bulge with the extended PSF. The result is shown in Figure 9. The $z$-profiles of our model are represented by solid lines, and the outer wings due to scattered light from the nucleus and bulge are represented by dashed

lines. We find that scattered light from the central region of NGC 4565 at least 2 mag $\operatorname{arcsec}^{-2}$ fainter than the model fit everywhere, including on the minor axis.

To test the effect of scattered light from the galaxy as a whole, we convolved the model with the full PSF (dotted line in Figure 9). As is evident, the extended PSF has little effect on the model we derive for the faintest parts of NGC 4565, being at most 0.2 - 0.3 mag different at very faint surface brightness, where 
Table 6: parameters of the thin-disk + thick-disk + power-law halo model.

\begin{tabular}{lll}
\hline \hline Thin disk & Thick disk & Power-law halo \\
\hline$\mu_{0}=22.32_{+0.08}^{-0.04} \mathrm{mag} \mathrm{arcsec}^{-2}$ & $\mu_{0}=25.52_{+0.41}^{-0.50} \mathrm{mag} \mathrm{arcsec}^{-2}$ & $\mu_{0}=26.86_{+0.86}^{-0.64} \mathrm{mag} \operatorname{arcsec}^{-2}$ \\
$z_{0}=1.17_{+0.04}^{-0.04} \mathrm{kpc}$ & $z_{0}=2.55_{+0.22}^{-0.30} \mathrm{kpc}$ & $r_{0}=14.44_{+4.74}^{-4.07} \mathrm{kpc}$ \\
$h_{r}=8.05_{+0.34}^{-0.19} \mathrm{kpc}$ & $h_{r}=11.03_{+1.12}^{-1.88} \mathrm{kpc}$ & $\gamma=3.88_{+0.56}^{-0.31}$ \\
$r_{\text {max }}=31.84_{+0.07}^{-0.12} \mathrm{kpc}$ & $r_{\text {max }}=36.93_{+0.22}^{-0.64} \mathrm{kpc}$ & $q=0.44_{+0.02}^{-0.02}$ \\
$\left(m_{6660}\right)^{a}=8.99 \pm 0.02 \mathrm{mag}$ & $\left(L_{\text {thick }} / L_{\text {thin }}\right)^{b}=20 \%$ & $\left(L_{\text {halo }} / L_{\text {thin }}\right)^{c}=21.4 \%$ \\
\hline
\end{tabular}

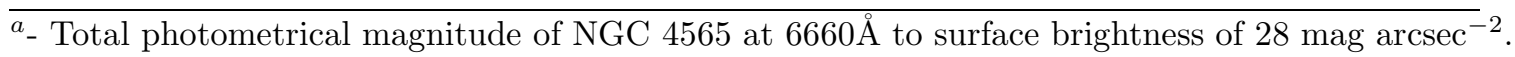

$b_{\text {- }}$ The luminosity ratio of model thick-disk and thin disk.

${ }^{c}$ - The luminosity ratio of model power-law halo and thin disk.

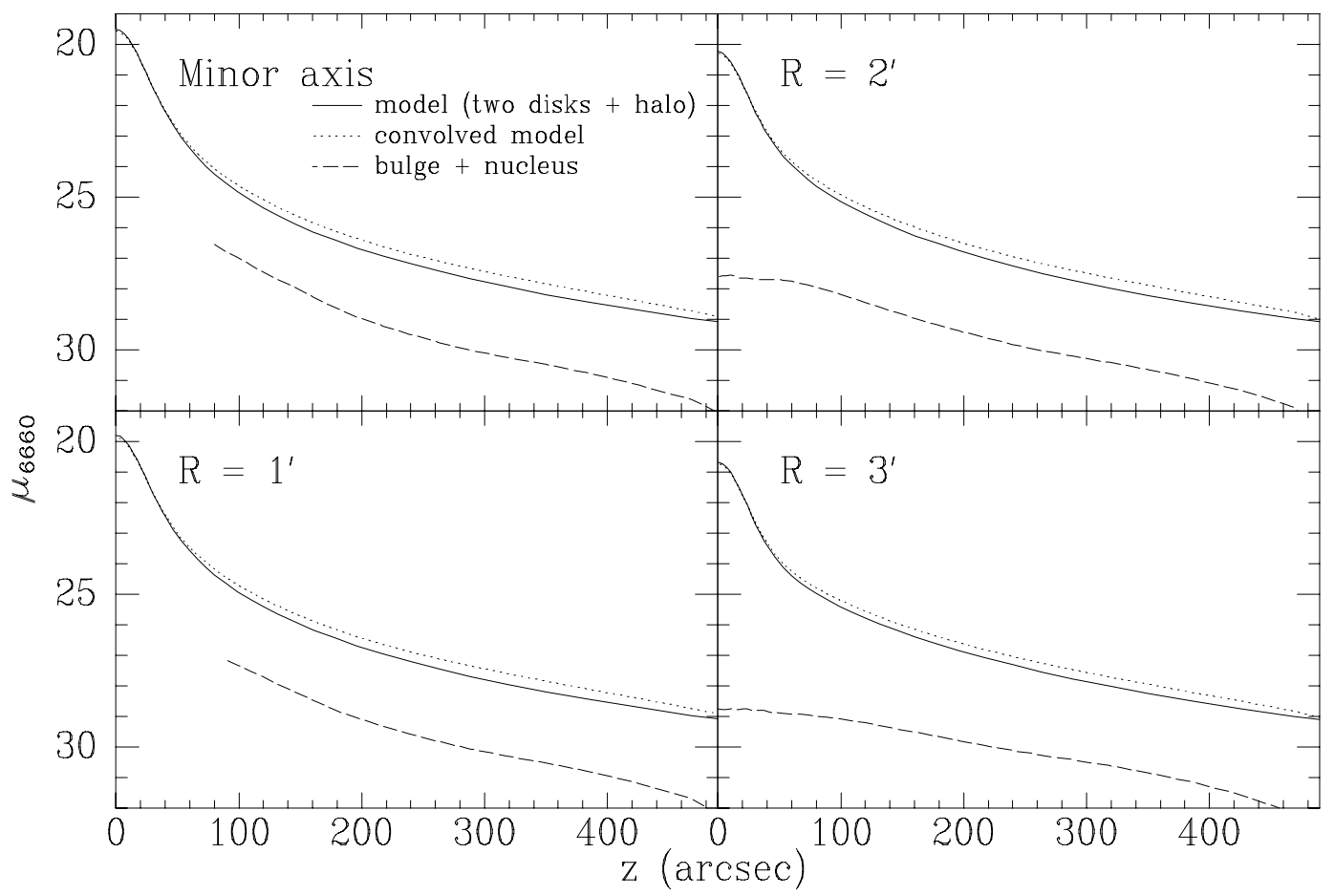

Fig. 9.- The possible effects of scattered light from the brighter parts of NGC 4565 on the model obtained from its faintest parts. The solid lines are our model, the dashed lines represent the scattered light from the nucleus and bulges, and the dotted lines are the model convolved with extended PSF. 
Table 7: A comparison of the parameters obtained from different sources.

\begin{tabular}{|c|c|c|c|c|c|c|c|c|}
\hline \multirow[t]{2}{*}{ component } & \multirow[t]{2}{*}{ filter } & \multirow{2}{*}{$\begin{array}{l}\text { thin-disk } \\
h_{r}(\mathrm{kpc})\end{array}$} & \multicolumn{3}{|c|}{ thick-disk } & \multirow{2}{*}{$\begin{array}{l}\text { third-disk(exp) } \\
\qquad h_{z}(\mathrm{kpc})\end{array}$} & \multirow{2}{*}{$\begin{array}{c}\text { or halo } \\
\qquad q\end{array}$} & \multirow[b]{2}{*}{$\gamma$} \\
\hline & & & $z_{0}(\mathrm{kpc})$ & $h_{r}(\mathrm{kpc})$ & $z_{0}(\mathrm{kpc})$ & & & \\
\hline $\mathrm{KS}$ & $\mathrm{B}_{\mathrm{J}}$ & 8.0 & 1.15 & & & & & \\
\hline H80 & B & 8.8 & & & & & 0.46 & 3.46 \\
\hline H82 & B & 9.7 & & & & & 0.50 & 4.10 \\
\hline JT & B & 9.9 & & 9.9 & 1.88 & 4.78 & 0.52 & \\
\hline R96 & $\mathrm{K}$ & 8.4 & $1.28^{a}$ & & & & & \\
\hline \multirow[t]{2}{*}{$\mathrm{SG}^{b}$} & B & 10.6 & 1.10 & 6.2 & 1.62 & 3.42 & & \\
\hline & $\mathrm{R}$ & 6.8 & 1.07 & 7.5 & 1.90 & 4.23 & & \\
\hline $\mathrm{NJ}^{b}$ & V & 8.1 & 0.87 & 8.6 & 1.88 & 5.1 & & \\
\hline \multirow[t]{2}{*}{$\mathrm{SG}^{c}$} & B & 8.0 & 1.20 & 12.9 & 2.28 & & 0.37 & \\
\hline & $\mathrm{R}$ & 7.0 & 1.13 & 9.1 & 2.29 & & 0.36 & \\
\hline $\mathrm{KB}$ & $\mathrm{V}$ & & & & & & & 3.46 \\
\hline SOSCB & $6040 \AA$ & & & & & & & 3.78 \\
\hline This work $^{d}$ & $6660 \AA$ & 8.05 & 1.17 & 11.03 & 2.55 & & 0.44 & 3.88 \\
\hline
\end{tabular}

${ }^{a}$ - R96 use sech disk model give $h_{z}=0.44$ which corresponding to $z_{0} / 2$.

$b^{b}-\operatorname{sech}^{2}+\operatorname{sech}^{2}+\exp$ model

${ }^{c}$ - $\operatorname{sech}^{2}+\operatorname{sech}^{2}+\mathrm{R}^{1 / 4}$ halo model

$d_{-} \operatorname{sech}^{2}+\operatorname{sech}^{2}+$ power-law halo model

Note.- All the values in the table are transformed to the distance of $14.5 \mathrm{Mpc}$.

KS: van der Kruit \& Searle 1981; H80: Hamabe et al. 1980; H82: Hamabe 1982; JT: Jensen \& Thuan 1982;

R96: Rice 1996; SG: Shaw \& Gilmore 1989; NJ: Näslund \& Jörsäter 1997; KB: Kormendy \& Bruzual 1978;

SOSCB: Spinrad et al. 1978 
the errors of measurement are at least that high. We conclude that, within the errors of measurement, our parameters for the halo of NGC 4565 are not significantly affected by scattered light from the brighter parts of the galaxy.

\subsection{Inclination}

We estimate the inclination of the disk of NGC 4565 by assuming that the thin disk of this galaxy is round with a radius of $38 \mathrm{kpc}$, and the dust lane is flat and lies at the mid-plane of the disk. We assume that the upper-edge (cf. Figure 4) of the dust-lane could be the edge of round disk. By measuring the projected distance between the upper-edge of the dust-lane and the major axis of NGC 4565 in the plane of the sky, taking into account the radius of the disk, we obtain an inclination of $87.5^{\circ}$, which is somewhat higher than that of $86^{\circ}$ in $\mathrm{KS}(1981)$.

Given our model assumes that the galaxy is exactly edge-on $\left(i=90^{\circ}\right)$, we can test how our model changes for different values of $i$ via the following transformation:

$$
\sigma(R, z)=\int_{\frac{-\sqrt{r_{\max }^{2}-R^{2}}-z \cos i}{\sin i}}^{\frac{\sqrt{r_{\max }^{2}-R^{2}}-z \cos i}{\sin }} \operatorname{sech}^{2}\left(\frac{|z \sin i-l \cos i|}{z_{0}}\right) \exp \left(\frac{-\sqrt{R^{2}+(z \cos i+l \sin i)^{2}}}{h_{r}}\right) d l .
$$

The variable of integration, $l$, is along the line-of-sight. We plot the minor axis profiles with $90^{\circ}, 87.5^{\circ}$, and $86^{\circ}$ for both the thin and the thick disks in Figure 10. As might be expected, the form of the thick disk is relatively insensitive to the exact inclination used for this galaxy. Even the thin disk model is only slightly more sensitive, having a central surface brightness $\sim 0.1-0.2 \mathrm{mag} \operatorname{arcsec}^{-2}$ fainter for $87.5^{\circ}$ than for $90^{\circ}$.

\subsection{Bulge}

The bulge can be obtained, after subtraction of the disks and halo. We show the two-dimensional distribution of the bulge and the profile along the minor axis in Figure 11. The bulge extends up to 5 $\mathrm{kpc}$, and it is clear that it cannot be described by $\mathrm{R}^{1 / 4}$-law (dashed line) from $0.7 \mathrm{kpc}$ to $5 \mathrm{kpc}$. Rather, an exponential distribution (cf. Kormendy \& Bruzual 1978) (solid line) with a scale height of $h_{z}=0.65$ $\operatorname{kpc}\left(\sim z_{0} / 2\right)$ is the best fit to the bulge luminosity distribution, consistent with the values of $0.72 \mathrm{kpc}$ determined by JT and $0.75 \mathrm{kpc}$ in the K-band (Rice 1996). We find the bulge to have an ellipticity of 0.8 , based on a simple fit using elliptical isophotes.

Speculation abounds in the literature about the nature of the bulge seen in NGC 4565. Is it bar-shaped, seen with the long axis projected along our line-of-sight (much like the bar of our own galaxy; cf. Blitz \& Spergel 1991)? Or is it dynamically coupled to the thin disk (which has a similar exponential scale height) 


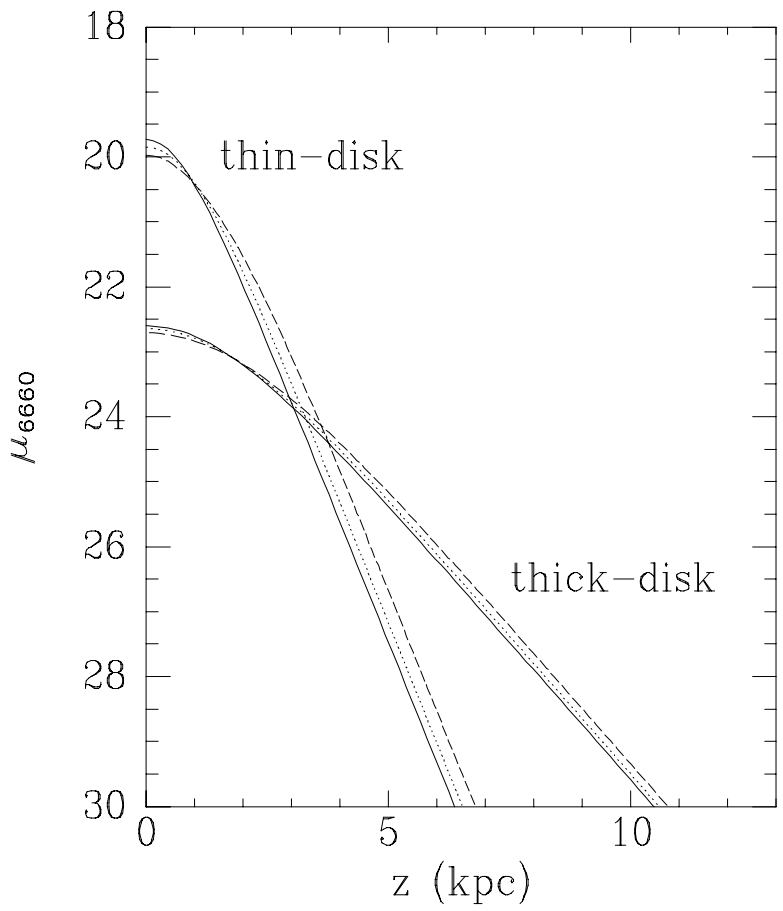

Fig. 10.- The effect of inclination on the model profiles of the minor axis of the thin and thick disks. Three inclinations are considered: $90^{\circ}$ (solid lines), $87.5^{\circ}$ (dotted lines) and $86^{\circ}$ (dashed lines). 
so that it takes a peanut-shaped or boxy shape? In absence of clear-cut dynamical data on the motions of stars in the bulge, the photometric data alone cannot choose among these options. Even so, the nearly pure exponential nature of the bulge of NGC 4565 hints more of a bar than it does of a typical $\mathrm{R}^{1 / 4}$-law bulge.

\subsection{Face-On Warp?}

The striking image presented of the bulge in Figure 11 highlights an issue that also can be seen in sky-subtracted image of the whole galaxy in Figure 4: there seems to be far fewer faint objects in the lower SW side of the galaxy disk than in the upper NE side of the galaxy disk. As seen in Figure 4, this asymmetry in number of giant objects seems to extend to 2-3 arcmin in the SW direction. Unfortunately, we cannot appeal to HST observations of this galaxy, as such imaging (Kissler-Patig, et al. 1999) avoided the NE side owing to the bright star there.

The only reasonable interpretation we can give to the existence of an asymmetry in the faint objects around NGC 4565 is that there is obscuring matter between NGC 4565 and the background galaxies. If this proves to be true (and we plan to investigate this issue further in another paper), then it could be evidence for a face-on warp in this galaxy. Such a warp would have to be part of the disk facing away from us, as the nucleus of this galaxy is obviously not obscured.

\section{SUMMARY}

We have obtained deep intermediate-band $(6660 \AA)$ surface photometry of the nearby, bright, edge-on galaxy NGC 4565. The combination of having a nearly 1 square degree field of view, the ability to use the dome to obtain high S/N flat fields, accurate subtraction of the PSF wings of stars, and an accurate method for two-dimensional sky subtraction combine to yield a limiting magnitude of 28.77 mag $\operatorname{arcsec}^{-2}$ (at which the observational error reaches $0.75 \mathrm{mag} \operatorname{arcsec}^{-2}$; or $0.25 \mathrm{mag}$ at a surface brightness of 27.5

mag $\operatorname{arcsec}^{-2}$ ). The sky background in our image is $20.72 \mathrm{mag} \operatorname{arcsec}^{-1}$, about $0.5 \mathrm{mag} \operatorname{arcsec}^{-2}$ brighter than the sky found in our NGC 5907 observations in the same filter (cf. Zheng et al. 1999). The total magnitude of NGC 4565 in $6660 \AA$-band is $8.99 \pm 0.02 \mathrm{mag}$ (equivalent to $\mathrm{R}=9.10$ ) to a surface brightness of $28 \mathrm{mag} \operatorname{arcsec}^{-2}$.

The luminosity distribution of the galaxy is presented in a series of cuts both parallel to its minor axis ( $z$-profiles) and parallel to its major axis ( $R$-profiles). Excluding the dust lane, the $z$-profiles are symmetric in all four quadrants out to a radius of 6 arcmin. The $z$-profiles extend to nearly $30 \mathrm{kpc}$ (at $29 \mathrm{mag}$ $\operatorname{arcsec}^{-2}$ ), farther than given by previous studies of this galaxy.

We construct a two-dimensional model of the outer parts of this galaxy comprised of three components 

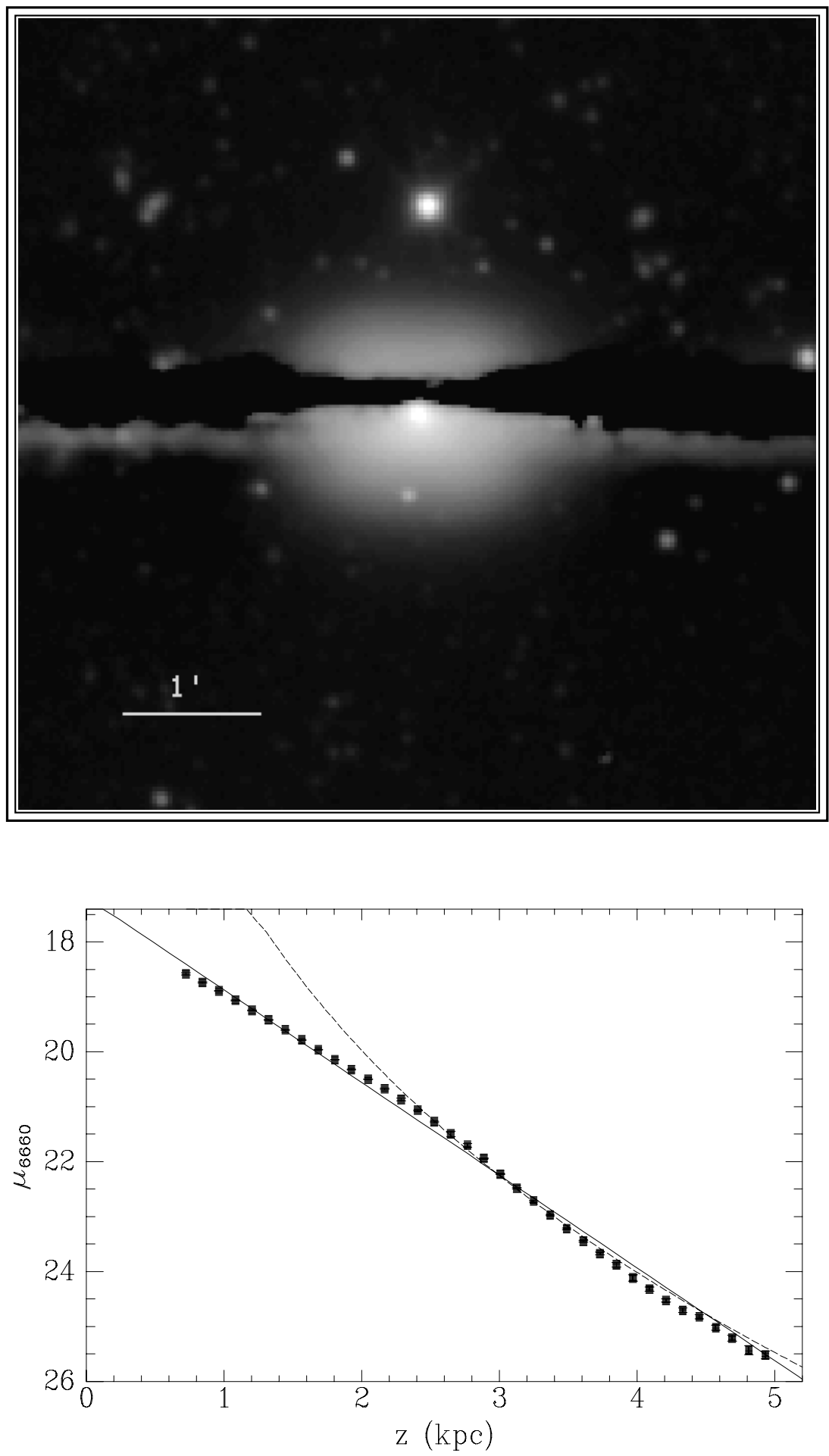

Fig. 11. - Top: The image of the bulge of NGC 4565 produced by subtracting the two-disk-halo model from the final image. The faint stellar images around the bulge are likely globular clusters in NGC 4565 . Bottom: The minor axis profile (solid boxes) of the bulge in NGC 4565 so-obtained. The solid line represents exponential model with the scale height $0.65 \mathrm{kpc}$. The dashed line is the $\mathrm{R}^{1 / 4}$ law that best fits the outer part of the bulge. The bulge of NGC 4565 is fit better by an exponential model than an $\mathrm{R}^{1 / 4}-$ law model. 
(thin-disk + thick-disk + halo). Altogether the model includes 12 parameters, including a cut-off radius for the disk. The values of these parameters are determined by the $\chi^{2}$ values from a series of 3,700 initial starting values which eventually converge to distinct values. The parameters so-derived for the thin and thick disks generally agree with those derived by previous studies. As our halo observations go much deeper than those of previous studies, we obtain a more reliable measurement of the power-law behavior of the halo, with its power-law index $\gamma$ determined to lie between 3.2 and 4.0 (best-fit value of 3.88). Our observations effectively rule out the possibility of an $r^{-2}$ halo in NGC 4565 . We obtain an axis ratio for the halo of this galaxy to be equal to 0.44 and core radius of $14.4 \mathrm{kpc}$, which suggest a flattened ellipsoid.

We test for two factors which could affect our results: scattered light from the galaxy itself and an incorrect value used for the inclination of the galaxy disk. We find neither significantly affects our results. However, if our data are accurate enough, the effects would be present, at most, at the $0.2-0.3 \mathrm{mag} \operatorname{arcsec}^{-2}$ level. We measure an inclination of NGC 4565 of $i=87.5^{\circ}$ from the offset of the dust lane in the inner disk from the nucleus of the galaxy.

We obtain a two-dimensional luminosity distribution for the bulge of NGC 4565 by subtracting our two-disk+halo model from the original image. The luminosity profile along the minor axis of the bulge of NGC 4565 is well fitted by exponential model with scale height similar to that of the thin disk (consistent with was has been found from previous studies).

In the coming years, our survey will obtain deep images in all of the 15 BATC passbands (cf. Zhou et al.) of NGC 4565, as well as for a number of other, nearby galaxies. With a complete sampling of the visible and near-infrared spectrum of these galaxies, we will be able to study the stellar populations in their disks, bulges and halos.

We thanks Professors Qiuhe Peng, Dr. Xiangping Wu and Dr. Shude Mao for valuable discussion. Also many thanks to Dr. Jianyan Wei. The BATC Survey is supported by the Chinese Academy of Sciences (CAS), the Chinese National Natural Science Foundation (CNNSF) and the Chinese State Committee of Sciences and Technology (CSCST). We also thank the Chinese National Pandeng Project for financial support. The project was also supported in part by the U.S. National Science Foundation (NSF Grant INT-93-01805), by Arizona State University, the University of Arizona, and Western Connecticut State University. 


\section{REFERENCES}

Bertin, E., \& Arnouts, S. 1996, A\&AS, 117, 393

Blitz, L. \& Spergel, D. 1991, ApJ,379, 631

Burstein, D. 1979, ApJ, 234, 829

de Grijs, R., \& van der Kruit, P. C. 1996, A\&AS, 117, 19

Dettmar, R. J., \& Wielebinski, R. 1986, A\&A, 167, L21

de Vaucouleurs, G., de Vaucouleurs, A., Corwin, H.C.G., Jr., Buta, R. J., Paturel, G. \& Fouqué, P. 1991, Third Reference Catalog of Bright Galaxies (New York: Springer-Verlag)

Fan, X.-H. et al. 1996, AJ, 112, 628

Freedman, W.L, 2001, ApJ, in press

Fry, A. M., Morrison, H. L., Harding, P., \& Boroson, T. A. 1999, AJ, 118, 1209

Hamabe, M., Kodaira, K., Okamura, S., \& Takase, B. 1980, PASJ, 32, 197 (H80)

Hamabe, M. 1982, PASJ, 34, 423 (H82)

Ho, L. C., et al. 1997, ApJS, 112, 391

Jensen, E. B., \& Thuan, T. X. 1982, ApJS, 50, 421 (JT)

Kissler-Patig, M., Ashman, K.M., Zept, S.E., \& Freeman, K.C. 1999, AJ, 118, 197

Kormendy, J., \& Bruzual, A. G. 1978, ApJ, 223, L63 (KB)

Liu, Y., 2001, thesis for Bachelor Degree, Nanjing University

Morrison, H. L., Boroson, T. A., \& Harding, P. 1994, AJ, 108, 1191 (MBH)

Näslund, M., \& Jörsäter, S. 1997, A\&A, 325, 915 (NJ)

Odewahn, S.C., Windhorst, R.A., Driver, S.P., Keel, W.C. 1996, ApJ, 472, 13

Oke, J. B., \& Gunn, J. E. 1983, ApJ, 266, 713

Press, W. H. et al. 1992, Numerical Recipes in Fortran, Second Edition (Cambridge University Press, Cambridge) 
Pritchet, C. J., \& van den Bergh, S. 1994, AJ, 107, 1730

Rice, W. 1996, AJ, 112, 114 (R96)

Sackett, P. D., Morrison, H. L., Harding, P., \& Boroson, T. A. 1994, Nature, 370, 441

Shang, Z. H., et al. 1998, ApJ, 504, L23

Sancisi, R. 1976, A\&A, 53, 159

Shaw, M. A., \& Gilmore, G. 1989, MNRAS, 237, 903 (SG)

Spinrad, H., Ostriker, J. P., Stone, R. P. S., Chiu, L. T. G., \& Bruzual, A. G. 1978, ApJ, 225, 56 (SOSCB)

Tonry, J., \& Schneider, D. P. 1988, AJ, 96, 807

van der Kruit, P. C. 1979, A\&AS, 38, 15

van der Kruit, P. C., \& Searle, L. 1981, A\&A, 95, 105 (KS)

Wild, W. J. 1997, PASP, 109, 1269

Willick, J. A., et al. 1996, ApJ, 457, 460

Yan, H., et al. 2000, PASP, 112, 691

Zheng, Z. Y., et al. 1999, AJ, 117, 2757

Zinn, R. 1985, ApJ, 293, 424

Zhou, X., et al., in preparation

This manuscript was prepared with the AAS $\mathrm{LAT}_{\mathrm{E}} \mathrm{X}$ macros v4.0. 
This figure "fig1.gif" is available in "gif" format from: http://arxiv.org/ps/astro-ph/0111433v1 
This figure "fig2.gif" is available in "gif" format from: http://arxiv.org/ps/astro-ph/0111433v1 
This figure "fig4.gif" is available in "gif" format from: http://arxiv.org/ps/astro-ph/0111433v1 
This figure "fig5a.gif" is available in "gif" format from: http://arxiv.org/ps/astro-ph/0111433v1 
This figure "fig5b.gif" is available in "gif" format from: http://arxiv.org/ps/astro-ph/0111433v1 
This figure "fig8.gif" is available in "gif" format from: http://arxiv.org/ps/astro-ph/0111433v1 